\title{
Original
}

\section{Calidad de vida relacionada con la salud en trabajadores sanitarios}

\author{
Health related quality of life in health workers
}

\section{Paloma Burgos Díez', Tomás Ruiz Albi ${ }^{2}$, Daniel Queipo Burón ${ }^{3}$, Fernando Rescalvo Santiago', María Mercedes Martínez León ${ }^{3}$, Piedad del Amo Merino', Carolina Burgos Díez ${ }^{4}$}

1. Servicio de Prevención de Riesgos Laborales. Hospital Universitario "Río Hortega". Valladolid. España.

2. Servicio de Neumología. Hospital Universitario "Río Hortega". Valladolid. España. Hospital Clínico Universitario. Valladolid. España.

3. Universidad de Valladolid. España.

4. ABS Apenins. Badalona (Barcelona). España.

Recibido: 13-12-11

Aceptado: 10-01-12

\section{Correspondencia}

Paloma Burgos Díez

Hospital Universitario "Río Hortega"

Dulzaina, 2

47012 Valladolid. España.

Tfno: 619252969

E-mail:palomaburgos@ono.com

Resumen

El estado de bienestar en que vivimos en los países desarrollados hace que surja la necesidad de buscar herramientas que sean una imagen fiel del estado de salud de la población como es la medición de la calidad de vida relacionada con la salud.

El objetivo de nuestro estudio es conocer la calidad de vida relacionada con la salud (CVRS) de los trabajadores sanitarios como medida de su estado de salud.

Para ello hemos realizado un estudio transversal, prospectivo y descriptivo de una muestra de 542 trabajadores seleccionada por muestreo sistemático. Como variable dependiente se evaluó la CVRS a través del cuestionario SF-36. Este cuestionario evalúa el estado de salud a través de 7 escalas (Función física, rol físico, salud general, vitalidad, función social, rol emocional y salud mental) valoradas de 0 a 100 (siendo 100 el mejor valor) y de dos dominios globales estandarizados para población general siendo su valor 50 , (índice general de salud física e índice general de salud mental). Se tiene en cuenta la edad y el sexo para el análisis de los resultados.

Los resultados más relevantes obtenidos son: Edad media 46 años (DE 10,4), 82\% mujeres. Función física: 88.7 (Población general-PG-: 84.7); Rol físico: 86.5 (PG: 83.2); Dolor: 71.9 (PG: 79); Salud general: 70 (PG: 68.3); Vitalidad: 66.1 (PG: 66.9); Función social: 86.4 (PG: 90.1); Rol emocional: 87.8 (PG: 88.6); Salud mental: 72.6 (PG: 73). Índice global de salud física: 51.9; Índice global de salud mental: 49.8.

Los valores encontrados tanto para las escalas de salud física como mental son ligeramente mejores que los establecidos para la población general para el mismo rango de edad y sexo, salvo en la escala de dolor.

Med Segur Trab (Internet) 2012; 58 (226) 27-34

Palabras clave: Calidad de vida relacionada con la salud. Trabajadores sanitarios. Estado de salud de trabajadores sanitarios; SF-36. 


\section{Abstract}

||||||||||||||||||||||||||||||||||||||||||||||||||||||||||||||||||||||||||||||||||||||||||||||||||||||||||||||||||||||||||||||||||||||||||||||||||||||||||||||||||||||||||||||||||||||||||||||||||||||||||||||||

The welfare state in whom we live in the developed countries does that there arises the need to look for tools that are a faithful image of the health of the population such as it is the measurement of the quality of life related to the health.

The aim of our study is to know the health related quality of life (HRQOL) of the sanitary workers as measure of their health status. For it we have realized a cross-sectional, prospective and descriptive study of a sample of 542 workers selected by systematic sampling.

The dependent variable was assessed HRQOL by the SF-36. This questionnaire assesses the health status across 7 scales (physical function, role physical, general health, vitality, social function, emotional role and mental health) from 0 to 100 (100 being the best value) and two global domains standardized for general population value being 50, (general index of physical health, and general index of mental health). It takes into account the age and the sex for the analysis of the results.

The most important results obtained are: Middle Age 46 years (SD 10,4), 82 \% women. Physical function: 88.7 (PG-general population: 84.7), role physical, 86.5 (PG: 83.2); Pain 71.9 (PG 79), general health: 70 (PG: 68.3); Vitality: 66.1 (PG: 66.9 ), social function: 86.4 (PG: 90.1), Role emotional 87.8 (PG: 88.6); Mental Health: 72.6 (PG 73).

Global index of physical health: 51.9; global Index of mental health: 49.8. The values found so much for the scales of physical as mental health are lightly better than the established ones for the general population for the same range of age and sex, except in the scale of pain.

Med Segur Trab (Internet) 2012; 58 (226) 27-34

Keywords: Health related Quality of life (HRQOL). Health workers. State of health of sanitary workers. $S F-36$. 


\section{INTRODUCCIÓN}

La preocupación por la salud ha sido siempre un motivo de interés desde el comienzo de la humanidad. Es un concepto que ha ido evolucionando dependiendo de los distintos contextos históricos, culturales y nivel de conocimientos desde una concepción mágicoreligiosa de la salud, al momento actual donde con los avances científicos y tecnológicos y sus consecuencias como el incremento de los procesos crónicos o la mejora para hacer frente a los procesos agudos, se hace indispensable valorar la salud desde otra perspectiva que no sea sólo la ausencia de enfermedad.

Con estas premisas la OMS en su Carta Constitutiva de 1946 propone dar un nuevo enfoque al concepto de salud al definirlo como "el más completo estado de bienestar físico, mental y social .y no solo la ausencia de enfermedad".

Teniendo en cuenta este concepto de salud como bienestar global, se ha desarrollado de forma paralela, el concepto de calidad de vida y en concreto su variante relacionada con la salud como una forma de valorar la percepción del estado de salud individual.

Tradicionalmente la medición del estado de salud de los individuos se había realizado a través de índices objetivos, que no eran una imagen fiel de la situación real, ya que no medían la calidad de vida o estado de salud individual según la concepción planteada por la OMS. A mediados de los años 70 se desarrollan nuevos instrumentos que consiguen dar esa visión conjunta del estado de salud. Entre otros, aparecen cuestionarios específicos para problemas determinados de salud, ventajosos como discriminativos aunque adolecen de dar una visión general de la salud (Rótterdam, EORT, escala de McGill...); o cuestionarios genéricos que son empleados para todo tipo de situaciones y miden múltiples dimensiones o categorías y se aplican a una amplia muestra poblacional, como el Nottingham, el SIP, o el SF-36 que es el que hemos utilizado en nuestro estudio por considerarlo como la mejor herramienta disponible para medir el estado de salud de una población desde un punto de vista global como es la salud de nuestros trabajadores.

Desde el origen del hombre y ante la necesidad de alimentarse y sobrevivir, ha existido el trabajo y con el, se comenzaron a producir los accidentes, las enfermedades derivadas del mismo e incluso la muerte.

Es por ello una necesidad la búsqueda de la salud desde el punto de vista de la calidad de vida como un concepto imbricado al trabajo. Los modelos de producción, la industrialización, la especialización, son unas de las variadas complicaciones con las que nos encontramos a la hora de conceptuar la calidad de vida; la necesidad de mantener unas óptimas condiciones de vida para los trabajadores es comúnmente aceptada por los empleadores, los trabajadores y la sociedad ${ }^{1}$.

Sin embargo, junto a esta influencia positiva del trabajo respecto a la salud, existe también una influencia potencialmente negativa: trabajando se puede perder la salud cuando el trabajo se desarrolla en condiciones que puedan causarnos daño. Igualmente, cuando el trabajo no es capaz de utilizar las máximas capacidades de un trabajador se corre el peligro de no permitir su desarrollo y realización como miembro de la sociedad.

La Encuesta Nacional de condiciones de Trabajo, revela datos preocupantes sobre la afectación subjetiva que tiene el trabajo sobre la salud de los empleados, que en el caso del sector sanitario ascendía a un $28 \%^{2,3}$

En los últimos años, el trabajo del personal sanitario se ha visto alterado por diversos factores, algunos de nueva aparición que se suman a los clásicos ya conocidos. El incremento de las enfermedades crónicas, los movimientos migratorios o el envejecimiento de la población han contribuido a estos cambios. Esto unido a las limitaciones en el tiempo de atención al paciente, hace que pueda suponer una amenaza para la calidad de vida relacionada con la salud de nuestros trabajadores y por ello la necesidad de realizar estudios dirigidos a buscar como repercuten estas situaciones en su salud global. 


\section{OBJETIVOS}

Hemos elaborado un estudio que nos permita conocer la CVRS en los trabajadores del ámbito sanitario como una medida de su estado de salud, para luego establecer una comparación con los resultados obtenido a través de la misma medida y para la población general.

\section{SUJETOS Y MÉTODO}

Para la consecución de los objetivos enumerados se llevó a cabo el siguiente diseño:

Se trata de un estudio transversal, prospectivo, descriptivo y analítico de los trabajadores de dos provincias castellano-leonesas. Las características de los trabajadores de ambas áreas eran similares para las variables principales.

Se realizó un muestreo sistemático de los trabajadores de ambas áreas con un tamaño muestral de 542 trabajadores.

El tamaño muestral fue calculado para esperar una diferencia de 3 puntos en la escala normalizada del cuestionario SF-36 que pudiese tener repercusión en la clínica, con lo que la n esperada era de 350 sujetos, nosotros añadimos más trabajadores al estudio para aumentar la potencia de los resultados ${ }^{4}$.

El cuestionario de salud SF-36 es un cuestionario de amplia distribución mundial fue desarrollado para medir conceptos genéricos de salud tanto física como mental aplicable a pacientes o a población general. La versión española fue publicada por Alonso y colaboradores en 19955. Desde entonces se ha utilizado en un gran número de publicaciones y artículos.

Proporciona un método exhaustivo y psicométricamente sólido, destacado como uno de los instrumentos más útiles en la evaluación de la CVRS, como ha mostrado en el análisis sistemático realizado por Villagut en 2005 sobre el uso del cuestionario en España ${ }^{6}$.

Se trata de un cuestionario autoadministrado con presencia de entrevistador, que está compuesto por 36 preguntas o ítems que cubre 8 dimensiones.

Las dimensiones del cuestionario nos facilitan unas puntuaciones que son directamente proporcionales al estado de salud entre 0 y 100 de peor a mejor estado de salud. (Los ítems se codifican, se agregan y transforman en una escala con recorrido de 0 a 100.)

El cuestionario permite dos puntuaciones sumario que son el índice global de salud física y el índice global de salud mental, obtenidos a través de la combinación de las puntuaciones de cada dimensión. Y que sus valores están estandarizados para la población de referencia que presenta una media de 50.

Se ha realizado un análisis estadístico descriptivo mediante la media, mediana, desviación estándar, percentiles y amplitud intercuartil para las variables cuantitativas y las frecuencias para las variables categóricas. Se calcula el intervalo de confianza al 95\% para la población.

En el análisis se han utilizado pruebas de correlación entre variables cuantitativas y T-Test para comparación de medias entre los sexos y con la población general.

\section{RESULTADOS}

Destacar respecto a los resultados expuestos que la edad media y el porcentaje de trabajadores por sexos es similar a lo descrito para el resto de centros sanitarios de la Comunidad $^{7}$; en nuestro estudio la edad media de los trabajadores fue de 46 años (DE 10,4) siendo un $82 \%$ del sexo femenino, sin encontrarse diferencias por edad respecto al sexo. 


\section{Resultados generales}

En la tabla I se muestran los resultados descriptivos del cuestionario Sf 36.

Las dimensiones que mostraron valores más altos fueron la función física (88.7) y el rol emocional (87.8) y las que mostraron peores resultados la vitalidad (66.1) y la salud general (70).

En cuanto a los índices globales vemos valores superiores en la salud física que en la mental (51.9 vs 49.8)

Tabla I

\begin{tabular}{lcccccc}
\hline \multicolumn{1}{c}{ Dimensiones } & Media & DE & Rango & Mediana & Amplitud intercuartil & IC 95\% \\
\hline Función física: & 88,7 & 15,3 & $20-100$ & 95 & $85-100$ & $87,4-90$ \\
Rol físico: & 86,5 & 30,5 & $0-100$ & 100 & $100-100$ & $83,9-89,1$ \\
Dolor: & 71,9 & 24,1 & $0-100$ & 72 & $52-100$ & $69,9-74$ \\
Salud general: & 70 & 16,2 & $20-100$ & 72 & $57-82$ & $68,6-71,3$ \\
Vitalidad: & 66,1 & 18,5 & $0-100$ & 70 & $55-80$ & $64,6-67,7$ \\
Función social: & 86,4 & 19,7 & $12,5-100$ & 100 & $75-100$ & $84,7-88,1$ \\
Rol emocional: & 87,8 & 28,6 & $0-100$ & 92 & $100-100$ & $85,4-90,3$ \\
Salud mental: & 72,6 & 18,4 & $9-100$ & 77,2 & $59-86$ & $71-74,1$ \\
\multicolumn{1}{c}{ ISF* } & 51,9 & 8,7 & $14,3-72,7$ & 54 & $48-57$ & $51,1-52,6$ \\
\multicolumn{1}{c}{ ISM** } & 49,8 & 11,2 & $6-68$ & 53 & $45-57$ & $48,9-50,8$ \\
\hline (́ndice global de salud física. * Índice global de salud mental & & & \\
\hline
\end{tabular}

Cuando analizamos los resultados del cuestionario por edad y sexo (tabla II), hemos podido observar que todas las dimensiones presentan una correlación negativa con la edad, siendo más importantes y significativas las dimensiones físicas (función física, rol físico, dolor y salud general). Lo que se evidencia en los resultados de los índices globales $(\mathrm{p}<0.001)$.

Respecto al sexo, si comparamos las medias de los resultados observamos que las mujeres presentan valores inferiores a los varones, siendo estas diferencias más significativas en la esfera mental (ISM 49 vs 53.6; $\mathrm{p}<0.001$ ).

Tabla II

\begin{tabular}{lccccc}
\hline & \multicolumn{2}{c}{ Edad } & \multicolumn{2}{c}{ Sexo } \\
\cline { 2 - 5 } \multicolumn{1}{c}{ Dimensiones } & $\mathbf{R 2}$ & $\mathbf{p}$ & $\mathbf{H}$ & $\mathbf{M}$ & $\mathbf{p}$ \\
\hline Fun física & $-0,31$ & $<\mathbf{0 , 0 0 1}$ & 92,7 & 87,8 & $\mathbf{0 , 0 0 4}$ \\
Rol físico & $-0,12$ & $\mathbf{0 , 0 0 4}$ & 90,3 & 85,7 & 0,182 \\
Dolor & $-0,21$ & $\mathbf{0 0 , 0 0 1}$ & 78,2 & 70,6 & $\mathbf{0 , 0 0 5}$ \\
S. general & $-0,21$ & $\mathbf{0 0 , 0 0 1}$ & 73,8 & 69,1 & $\mathbf{0 , 0 1 1}$ \\
Vitalidad & $-0,09$ & $\mathbf{0 , 0 2 2}$ & 75,3 & 64,1 & $<\mathbf{0 , 0 0 1}$ \\
Fun social & $-0,11$ & $\mathbf{0 , 0 0 8}$ & 91,7 & 85,3 & $\mathbf{0 . 0 0 4}$ \\
Rol emocio & $-0,02$ & 0,533 & 93,1 & 86,7 & 0,042 \\
S. mental & $-0,08$ & 0,053 & 79,4 & 71 & $<\mathbf{0 0 1}$ \\
\multicolumn{1}{c}{ ISF* } & -0.25 & $<\mathbf{0 , 0 0 1}$ & 53,2 & 51,6 & 0,100 \\
\hline \multicolumn{1}{c}{ ISM** } & $-0,18$ & 0,682 & 53,6 & 49 & $<\mathbf{0 , 0 0 1}$ \\
\hline
\end{tabular}




\section{Respecto a población general}

Cuando comparamos nuestros resultados con los obtenidos para la población general española descritos por Alonso ${ }^{8}$ en una muestra de 9151 personas mayores de 18 años, podemos observar cómo obtenemos mejores resultados entre nuestros trabajadores (ver Tabla III).

Para ello hemos realizado ajustes de datos por sexo y hemos analizado de forma independiente los resultados de las mujeres y de los varones, vemos como en éstos últimos, la salud parece mejor que la población general con diferencias significativas en las dimensiones que hacen referencia a la esfera física, como el caso de la función física o del rol físico con diferencias de hasta 6 puntos en el sexo masculino.

En cuanto al colectivo femenino la distribución es similar, los resultados son mejores en función física, rol físico y salud general, sin embargo los valores son inferiores en la función social.

Tabla III

\begin{tabular}{|c|c|c|c|c|c|c|c|c|c|}
\hline & & \multicolumn{2}{|c|}{ Muestra } & \multicolumn{2}{|c|}{ P. general } & \multirow{2}{*}{ Dif. } & \multicolumn{2}{|c|}{ IC 95\% } & \multirow{2}{*}{ p } \\
\hline & & Med & DE & Med & DE & & sup & $\inf$ & \\
\hline \multirow{3}{*}{$\begin{array}{l}\text { Función } \\
\text { física }\end{array}$} & Tot & 88,8 & 15 & 84,7 & 24 & 4,1 & 2,7 & 5,4 & $<0,005$ \\
\hline & $\mathrm{H}$ & 92,7 & 13 & 88,2 & 21 & 4,5 & 1,8 & 7,1 & $<0,005$ \\
\hline & M & 87,9 & 15 & 81,5 & 25 & 6,4 & 4,8 & 7,9 & $<0,005$ \\
\hline \multirow{3}{*}{ Rol fisico } & Tot & 86,6 & 30 & 83,2 & 35 & 3,4 & 0,7 & 6 & 0,026 \\
\hline & $\mathrm{H}$ & 90,3 & 27 & 87,2 & 31 & 3,1 & $-2,3$ & 8,5 & 0,26 \\
\hline & M & 85,7 & 31 & 79,5 & 38 & 6,2 & 3,1 & 9,2 & $<0,005$ \\
\hline \multirow{3}{*}{$\begin{array}{l}\text { Dolor } \\
\text { corporal }\end{array}$} & Tot & 72 & 24 & 79 & 27 & -7 & $-4,8$ & $-9,1$ & $<0,005$ \\
\hline & $\mathrm{H}$ & 78,2 & 22 & 84 & 24 & $-5,8$ & $-1,3$ & $-10,2$ & 0,01 \\
\hline & M & 70,6 & 24 & 74,4 & 29 & $-3,8$ & $-1,4$ & $-6,1$ & $<0,005$ \\
\hline \multirow{3}{*}{$\begin{array}{l}\text { Salud } \\
\text { general }\end{array}$} & Tot & 70 & 16 & 68,3 & 22 & 1,7 & 0,2 & 3,1 & 0,019 \\
\hline & $\mathrm{H}$ & 73,8 & 14 & 70,8 & 21 & 3 & 0,1 & 5,8 & 0,04 \\
\hline & M & 69,2 & 16 & 65,9 & 22 & 3,3 & 1,6 & 4,9 & $<0,005$ \\
\hline \multirow{3}{*}{ Vitalidad } & Tot & 66,2 & 18 & 66,9 & 22 & $-0,7$ & 0,8 & $-2,2$ & 0,38 \\
\hline & $\mathrm{H}$ & 75,3 & 17 & 70,5 & 21 & 4,8 & 1,3 & 8,2 & 0,007 \\
\hline & M & 64,2 & 18 & 63.6 & 22 & 0,6 & $-1,1$ & 2,3 & 0,51 \\
\hline \multirow{3}{*}{$\begin{array}{l}\text { Función } \\
\text { social }\end{array}$} & Tot & 86,5 & 19 & 90,1 & 20 & $-3,6$ & $-1,9$ & $-5,2$ & $<0,005$ \\
\hline & $\mathrm{H}$ & 91,6 & 17 & 92,5 & 17 & $-0,9$ & 2,5 & $-4,3$ & 0,6 \\
\hline & M & 85,3 & 20 & 87,9 & 21 & $-2,6$ & $-0,6$ & $-4,5$ & 0,009 \\
\hline \multirow{3}{*}{ Rol social } & Tot & 87,9 & 28 & 88,6 & 30 & $-0,7$ & 1,8 & $-3,2$ & 0,57 \\
\hline & $\mathrm{H}$ & 93,2 & 22 & 92,9 & 24 & 0,4 & 4,8 & -4 & 0,85 \\
\hline & $\mathrm{M}$ & 86,7 & 29 & 84,4 & 34 & 2,3 & 5,1 & $-0,5$ & 0,11 \\
\hline \multirow{3}{*}{$\begin{array}{l}\text { Salud } \\
\text { mental }\end{array}$} & Tot & 72,6 & 18 & 73,3 & 20 & $-0,7$ & 0,8 & $-2,2$ & 0,38 \\
\hline & $\mathrm{H}$ & 79,4 & 16 & 76,9 & 18 & 2,5 & 5,7 & $-0,7$ & 0,13 \\
\hline & M & 71,1 & 18 & 70 & 20 & 1 & 2,7 & $-0,7$ & 0,27 \\
\hline
\end{tabular}

\section{DISCUSIÓN}

El cambio de las prioridades a lo largo de la historia ha ido modificando el significado del concepto de salud. Hasta ahora, la esperanza de vida ha sido un índice que se ha usado universalmente para comparar condiciones sociales, económicas y sanitarias entre los distintos países, ya que ha contado siempre con la ventaja de su independencia en la interpretación de la estructura por edad de la población de referencia. En los últimos años hemos vivido el incremento en la duración de la vida humana hasta alcanzar unos valores medios en nuestro país de 80,2 años, con clara diferencia positiva hacia el sexo femenino (84 en mujeres y 77 en hombres) ${ }^{9}$. 
A pesar de lo alentador de estos datos, no hay una total correspondencia con la percepción que tiene la población sobre su propio estado de salud, y esos años ganados en vida se pierden en incapacidad ${ }^{10}$.

Aceptando la dificultad de la medición de esta apreciación del sujeto de cómo vive la vida, y conociendo la necesidad y la utilidad de cuantificar los resultados, hemos seleccionado el que creemos es el cuestionario que mejor se podría adaptar a nuestros objetivos y a las características de nuestra muestra, es un cuestionario de amplia difusión a nivel mundial y bien adaptado a nuestra lengua y de excelente aplicabilidad a nuestro estudio, el SF-36.

Nuestro estudio versa sobre población sanitaria, y por tanto trabajadora, y en principio sin una limitación grave para sus quehaceres laborales, y refleja unos resultados, en esencia, similares a los publicados para la población general española, en lo que respecta a la tendencia por edad y sexo, aunque con valores medios absolutos superiores; parece que esto podría explicarse por el sesgo de "trabajador sano", ya que en la bibliografía está descrito que la población ocupada tiene mejor sensación de su estado de salud $^{11,12}$.

La tendencia de los resultados es igualmente equiparable a la de los resultados obtenidos en la población americana ${ }^{13}$. Las variaciones de resultados oscilan entre la escala de función física, que es la mejor valorada (88,7 de media en nuestra muestra; 84,7 en población general; 84,2 en EEUU) y la escala de vitalidad como la peor valorada $(66,1$ en nuestra muestra; 66,9 en población general). Lo que se evidencia en los resultados de los índices globales $(\mathrm{p}<0.001)$ y referido por Alonso ${ }^{5}$ en la población general en las 8 dimensiones del cuestionario y por Vilagut $^{6}$ en las dimensiones globales. Consideración que atribuyen en otros estudios a la edad, es decir, a mayor edad coexiste una mayor frecuencia de distintas patologías (publicación de AEMET).

Respecto a la función social que hace referencia a cómo los problemas de salud física o emocional interfieren en las actividades cotidianas, hay que destacar que un porcentaje de nuestros trabajadores realizan turnos de trabajo o incluso jornadas continuadas de 24 horas. Esto podría afectar a la valoración de esta escala.

Como resultado de haber llevado a cabo este trabajo, hemos podido observar y corroborar, que a pesar de que siempre son necesarias mejoras laborales y de que con el paso del tiempo y la evolución de la técnica hagan surgir nuevos riesgos y nuevas metas para conseguir eliminarlos, trabajamos en un medio adecuado en términos de calidad de vida relacionada con la salud.

\section{REFERENCIAS BIBLIOGRÁFICAS}

1. Casas J, Repullo J.R, Lorenzo S, Cañas J.J. Dimensiones y medición de la calidad de vida laboral en profesionales sanitarios. Revista de Administración Sanitaria (2002), 6, 23.

2. Declaración sobre la salud ocupacional para todos, aprobada en la segunda reunión de los Centros Colaboradores de la OMS para Salud Ocupacional Beijing, China 1994

3. VI Encuesta Nacional de Condiciones de Trabajo. Instituto Nacional de Seguridad e Higiene en el Trabajo (INSHT)- 2007.

4. Kazis y col consideran que diferencias inferiores a 0.2 veces la DE son pequeñas, 0.5 medianas y 0.8 grandes "Effect sizes for interpreting changes in health status". 1989. Medical care.

5. Alonso J, Prieto L, Antó JM. La versión española del SF-36 Health Survey (Cuestionario de Salud SF-36): un instrumento para la medida de los resultados clínicos. Med Clin 1995; 104: 771-6.

6. Vilagut G, Ferrer M, Rajmil L et al. El cuestionario de salud SF-36 español: una década de experiencia y nuevos desarrollos Gac Sanit. 2005; 19(2):135-150.

7. Estadística sobre establecimientos sanitarios con carácter de internado año 2007 y publicado por el MSC. Agencia de calidad del sistema Nacional de Salud 2007. Ministerio de Sanidad y Consumo. Disponible en: http://www.msc.es/en/novedades/docs/PlanCalidadSNS 
8. Alonso J, Regidor E, Barrio G, Prieto L, Rodriguez C, de la Fuente L. Valores poblacionales de referencia de la versión española del Cuestionario de Salud SF-36. Med Clin (Barc) 1998; 111: 410-416.

9. Estrategia de Desarrollo Sostenible de la UE. Publicada por INE. Disponible en: http://www.diariomedico. com/2009/05/20.

10. Gutiérrez JL, Regidor E. Esperanza de vida libre de incapacidad: un indicador global del estado de salud. Medicina clínica 1991; 96(12): 453-455.

11. Artazcoz L, Moya H, Venaclocha P, Pont P. La salud en el adulto. Gac. Sanit 2004; 18(supl 1):56-68.

12. P. Burgos Díez, P. del Amo Merino, T. Ruiz Albi, D. Queipo Burón, F. Rescalvo Santiago, Ma M. Martínez León, J. Burgoa Andrés. Influencia de las variables laborales en la presencia de morbilidad de los trabajadores sanitarios.

13. Ware JE, Snow KK, Kosinski M, \& Gandek B. SF-36 Health Survey manual and interpretation guide. Boston, MA: New England Medical Center, The Health Institute, 1993.

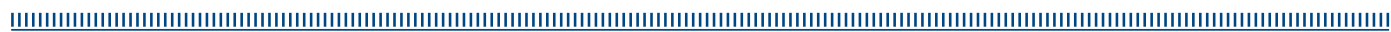

